\title{
QTL detection and allelic effects for growth and fat traits in outbred pig populations
}

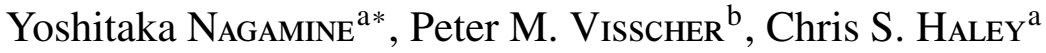 \\ ${ }^{\text {a }}$ Roslin Institute (Edinburgh), Midlothian, EH25 9PS, UK \\ ${ }^{\mathrm{b}}$ Institute of Cell, Animal and Population Biology, University of Edinburgh, \\ West Mains Road, Edinburgh, EH9 3JT, UK
}

(Received 17 February 2003; accepted 25 September 2003)

\begin{abstract}
Quantitative trait loci (QTL) for growth and fatness traits have previously been identified on chromosomes 4 and 7 in several experimental pig populations. The segregation of these QTL in commercial pigs was studied in a sample of 2713 animals from five different populations. Variance component analysis (VCA) using a marker-based identity by descent (IBD) matrix was applied. The IBD coefficient was estimated with simple deterministic (SMD) and Markov chain Monte Carlo (MCMC) methods. Data for two growth traits, average daily gain on test and whole life daily gain, and back fat thickness were analysed. With both methods, seven out of 26 combinations of population, chromosome and trait, were significant. Additionally, QTL genotypic and allelic effects were estimated when the QTL effect was significant. The range of QTL genotypic effects in a population varied from $4.8 \%$ to $10.9 \%$ of the phenotypic mean for growth traits and $7.9 \%$ to $19.5 \%$ for back fat trait. Heritabilities of the QTL genotypic values ranged from $8.6 \%$ to $18.2 \%$ for growth traits, and $14.5 \%$ to $19.2 \%$ for back fat. Very similar results were obtained with both SMD and MCMC. However, the MCMC method required a large number of iterations, and hence computation time, especially when the QTL test position was close to the marker.
\end{abstract}

QTL mapping / IBD / variance component / heritability / pig

\section{INTRODUCTION}

Following the first genome scan for QTL in $\mathrm{F}_{2}$ animals from a cross between wild boar and domestic pig [2,14], numerous studies were performed to locate QTL in pigs $[4,18,24]$. However, almost all the studies to date have focused on crosses (e.g., wild boar or Chinese breeds crossed with modern European breeds) because the power of detection of segregating QTL using line cross

\footnotetext{
* Corresponding author: nagamine@ affrc.go.jp

Present address: National Institute of Livestock and Grassland Science, 2 Ikenodai, Tsukuba, 305-0901, Japan
} 
data is greater than that using within population data $[13,30]$. In addition, it was uncertain whether detectable QTL would be segregating within a modern commercial pig population after their long selection history [29].

We previously reported significant QTL for growth and obesity detected using least squares (LS) analysis [17] on two chromosomes, 4 and 7, within five modern commercial pig populations [22]. The LS method is a widely used procedure and is as powerful as variance component analysis (VCA) in a simple pedigree structure [5]. However, the paternal half-sib LS method applied does not use potential information from segregation on the maternal side. Furthermore, another drawback of LS is that it may be used to estimate the differences between two allelic effects of QTL within a parent, but not the effect of each allele. On the other hand, VCA, using a model with polygenic and QTL effects, has some advantages for the detection of QTL. This procedure may be employed to estimate the polygenic and the QTL effect using information from the complete pedigree and VCA also allows the simultaneous estimation of heritabilities for both polygenic and QTL effects [1, 11, 21, 32]. Finally, each allelic effect of a QTL may be obtained, which provides direct information for marker-assisted selection [7].

George et al. [8] introduced a two-step approach for VCA. The first step involved the construction of an IBD matrix, while the next step was variance component estimation by restricted maximum likelihood to identify the QTL position. They applied the Markov chain Monte Carlo (MCMC) method using LOKI software [15] to estimate IBD coefficients. MCMC is a fairly flexible method, since it can handle pedigrees of any structure and cope with missing marker information. However, one major problem with this method is the computational time required. Nagamine et al. [21] and several researchers [23, 25] introduced the simple deterministic (SMD) method to estimate IBD coefficients for a population with a simple two-generation pedigree. This procedure is faster than MCMC and also copes with missing marker information

Following the determination of QTL within populations by LS analyses [22], we used the same dataset and applied both methods, SMD and MCMC, in VCA to estimate the QTL position for growth and fat traits. Furthermore, using the estimated QTL position, the QTL allelic effect and heritability were calculated. Using data from a separate study of different pig populations, de Koning and colleagues [5] used an alternative variance component approach for QTL detection. However, to our knowledge, this is the first report on the estimation of QTL genotypic and allelic effects, and simultaneous determination of heritabilities, polygene and the QTL genotypic effects within commercial pig populations. 


\section{MATERIALS AND METHODS}

\subsection{Data}

Within a total of 2713 animals, 576 Large White from population A, 580 Duroc-Large White from synthetic population B, 427 Yorkshire and Large White from synthetic population C, 531 Large White from population D and 599 Landrace from population $\mathrm{E}$ were genotyped. The numbers of sires, dams and progeny across the populations ranged from 10 to 12 , 91 to 178 , and 326 to 452 , respectively. Two growth traits, average daily gain on test (DGT) and average daily gain through whole life from birth to the end of test (DGW), and back fat thickness (BFT), were measured in the progeny generation. Population A additionally had phenotypic values from the parental generation. Standardized trait observations and weight, which was applied to adjust fat measurements, were used for a subsequent joint analysis of data from all five populations.

\subsection{Markers}

A maximum of eight and seven markers were genotyped on chromosomes 4 and 7, respectively. Specifically, the markers were S0001, SW45, SW35, SW839, S0107, S0217, SW841 and S0073 on chromosome 4, and SW1354, S0064, SWR1078, SW1344, TNFB, SW2019 and S0102 on chromosome 7. The numbers of genotyped markers ranged from 5 to 8 on chromosome 4 and 5 to 7 on chromosome 7 across the populations. Parents and progeny of all the populations were genotyped with both end markers, specifically, SO001 and S0073 on chromosome 4 (except population A), and SW1354 and S0102 on chromosome 7. Chromosome 4 from population A had 6 markers and SOOOI and $S W 841$ were used as the end markers. Selective genotyping was performed by identifying the $20 \%$ best and $20 \%$ worst progeny with respect to growth rate within the sire family. Data from all genotyped animals and the mapping software CRI-MAP [10] were used to confirm that no alternative marker orders were significantly better than the published consensus marker order (using the FLIPS option) and to estimate the distances between markers.

\subsection{Estimation of the IBD coefficient}

Two methods, MCMC and SMD, were employed to estimate IBD coefficients. The software, LOKI [15], was applied to estimate IBD coefficients with MCMC. SMD was developed by Nagamine et al. [21], based on the probability of inheriting alleles from a parental gamete [17]. If $p_{k}$ and $p_{j}$ represent the probability of inheriting the first paternal allele for animals $j$ and $k$ at a given location, the paternal allelic IBD coefficient (PA-IBD) between them is 
estimated with the following equation:

$$
\mathrm{PA}-\mathrm{IBD}=\left[p_{j} p_{k}+\left(1-p_{j}\right)\left(1-p_{k}\right)\right] / 2 .
$$

This is the genotypic IBD coefficient between half-sibs. The maternal allelic IBD (MA-IBD) coefficient is estimated similarly. The sum of PA-IBD and MA-IBD coefficients is taken as the genotypic IBD for full-sibs.

\subsection{Model and test statistics}

The following animal model was used:

$$
\mathbf{y}=\mathbf{X} \boldsymbol{\beta}+\mathbf{Z u}+\mathbf{Z w}+\mathbf{e}
$$

where the vector $\mathbf{y}$ represents the phenotypic values, $\mathbf{X}$ is the design matrix for fixed effect, and $\mathbf{Z}$ is the design matrix for random effects [16]. The remaining vectors are the estimated values, $\mathbf{u}$ : polygenic effect, w: QTL genotypic effect, e: error and $\beta$ : fixed effect. Sex was used as a fixed effect for growth traits and both sex and regression on weight were employed as fixed effects for fat traits. Variance components due to the random, polygenic and QTL genotypic effects were estimated by restricted maximum likelihood using ASReml software [9]. To estimate the presence of a QTL against the null hypothesis of no QTL at a test position, the likelihood ratio (LR) test statistic, $\log \mathrm{LR}=-2 \ln \left(\mathrm{L}_{0}-\mathrm{L}_{1}\right)$ was calculated, where $L_{0}$ and $L_{1}$ represent the respective likelihood values with the hypothesis, absence $\left(\mathrm{H}_{0}\right)$ or presence $\left(\mathrm{H}_{1}\right)$ of a QTL. The chi-square distribution with one degree of freedom $\left(\chi_{1}^{2}\right)$ was employed to provide approximate threshold values $[8,32]$. In the context in which it is used here, the distribution of LogLR for the test at a single point in the linkage group is a 50:50 mixture where one component mixture is a peak at 0 and the other is $\chi_{1}^{2}$. Therefore, the top $2 \%$ and $10 \%$ values from $\chi_{1}^{2}$ distribution, which represent approximately $1 \%$ and $5 \%$ point-wise significant levels of the $\mathrm{F}$ test, were used as threshold values.

\subsection{Conversion of QTL genotypic effect into allelic effect}

After estimating the QTL genotypic effect (w), we converted the values obtained into QTL allelic effects (v) [21]. Here, $\mathbf{G}$ is for allelic IBD matrix (size $2 n \times 2 n$ for $n$ animals), and $\mathbf{Q}$ is for genotypic IBD matrix (size $n \times n$ ). In case of no interaction between QTL allelic effects:

$$
\mathbf{w}=\mathbf{T v}
$$

where $\mathbf{T}$ is an incident matrix relating each animal to its two allelic effects $[20,27]$. This equation implies that the sum of two allelic effects from 
an animal, $v_{11}$ and $v_{12}$, is equivalent to his genotypic effect $w_{1}\left(=v_{11}+v_{12}\right)$. However, the conversion from $\mathbf{w}$ to $\mathbf{v}$ is less straightforward:

$$
\mathbf{v}=(1 / 2) \mathbf{G} \mathbf{T}^{\prime} \mathbf{Q}^{-1} \mathbf{w}
$$

Hence, $\mathbf{v}$ can be calculated without the inverse of $\mathbf{G}(2 n \times 2 n)$ and requires only the inverse of $\mathbf{Q}(n \times n)$. In fact, it is not necessary to calculate $\mathbf{Q}^{-1}$ for this conversion, since it is already obtained for use in mixed model equations to estimate $\mathbf{w}$.

\section{RESULTS}

\subsection{Marker distances}

The estimated marker distances (relative distance from the first marker: cM) were S0001 (0.0), SW45 (11.9), SW35 (11.9), SW839 (15.6), S0107 (17.1), S0217 (19.8), SW841 (23.9) and S0073 (28.4) on chromosome 4, and SW1354 (0.0), S0064 (6.4), SWR1078 (8.9), SW1344 (17.0), TNFB (27.5), SW2019 (29.3) and S0102 (39.3) on chromosome 7. These values are consistent with other published results (http://www.thearkdb.org/).

\subsection{Significant QTL effect}

The MCMC method employs an iterative approach to estimate IBD coefficients. Initially, test positions were spaced at 3 to $5 \mathrm{cM}$ intervals and 5000 iterations were used for each test positions. After identifying the regions with the higher test statistics, positions around these at $1 \mathrm{cM}$ intervals were examined. However, test positions within $1 \mathrm{cM}$ of the markers required more than 20000 iterations to produce an IBD matrix that was not singular.

With the LS method, five out of 26 combinations of trait, population and chromosome were significant at the nominal $1 \%$ level. All these combinations were also significant when estimated with both SMD and MCMC methods in VCA (Tab. I). However, combinations that were significant at the nominal 5\% level with LS were not usually significant in VCA. Only in one case (back fat depth on chromosome 4 in population C) were the VCA analyses significant when the LS analysis was not. The test statistic from the two VCA methods, SMD and MCMC, exhibited a high correlation of 0.95 (Fig. 1). The correlations of test statistic from LS and two VCA methods are 0.70 between LS and SMD and 0.71 between LS and MCMC. 


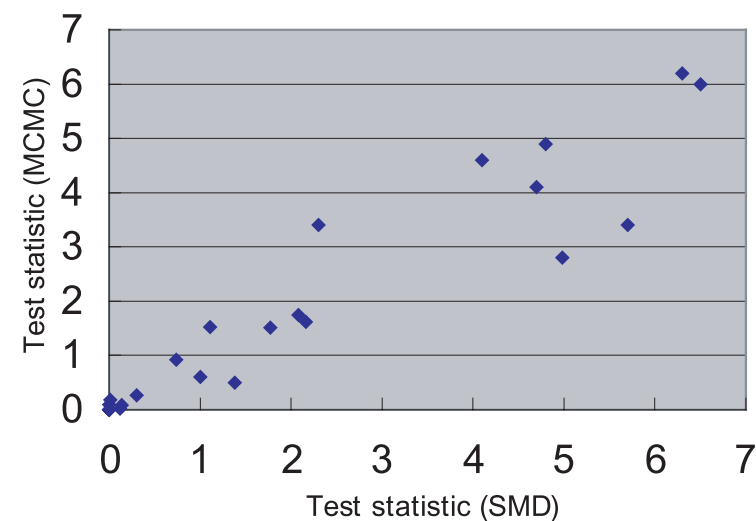

Figure 1. Test statistic (LogLR) from simple deterministic (SMD) and MCMC methods.

Table I. QTL test statistics for least squares and variance component analyses.

\begin{tabular}{|c|c|c|c|c|c|c|c|}
\hline \multirow[b]{2}{*}{ Population } & \multicolumn{4}{|c|}{ Chromosome 4} & \multicolumn{3}{|c|}{ Chromosome 7} \\
\hline & Trait & LS & SMD & MCMC & LS & SMD & MCMC \\
\hline \multirow[t]{2}{*}{ A } & DGT & 1.30 & 0.00 & 0.00 & $2.36 * *$ & $6.44^{++}$ & $6.00^{++}$ \\
\hline & BFT & 1.25 & 1.11 & 1.52 & $2.01 * *$ & $4.71^{+}$ & $4.09^{+}$ \\
\hline \multirow[t]{3}{*}{ B } & DGT & $1.99 *$ & 1.38 & 0.50 & 1.28 & 0.01 & 0.18 \\
\hline & DGW & $2.48 * *$ & $4.98^{+}$ & $2.80^{+}$ & 1.00 & 0.00 & 0.10 \\
\hline & BFT & $1.99 *$ & 1.77 & 1.51 & $1.86^{*}$ & 2.30 & $3.40^{+}$ \\
\hline \multirow[t]{3}{*}{$\mathrm{C}$} & DGT & 1.12 & 2.16 & 1.62 & 1.48 & 0.00 & 0.00 \\
\hline & DGW & 1.23 & 0.00 & 0.00 & 1.09 & 0.74 & 0.92 \\
\hline & BFT & 1.26 & $5.74^{++}$ & $3.41^{+}$ & $2.87 * *$ & $4.15^{+}$ & $4.56^{+}$ \\
\hline \multirow[t]{3}{*}{ D } & DGT & 0.81 & 0.00 & 0.00 & $1.87 *$ & 2.08 & 1.74 \\
\hline & DGW & 0.47 & 0.00 & 0.00 & $2.64 * *$ & $6.34^{++}-1-$ & $6.26^{++}$ \\
\hline & BFT & 1.11 & 0.14 & 0.08 & 1.74 & $4.88^{+}$ & $4.94^{+}$ \\
\hline \multirow[t]{2}{*}{$\mathrm{E}$} & DGT & 1.79 & 0.30 & 0.26 & 0.82 & 0.00 & 0.00 \\
\hline & BFT & 0.63 & 0.12 & 0.02 & $2.02 *$ & 1.00 & 0.60 \\
\hline
\end{tabular}

LS: least squares analysis; SMD: variance component analysis using simple deterministic method; MCMC: variance component analysis using MCMC method.

DGT: average daily gain on test; DGW: average daily gain of whole life from birth to end of test; BFT: back fat thickness.

** and *: significant with 1 and $5 \%$ level for $\mathrm{F}$ test in LS, respectively. ++ and +: significant with 2 and $10 \%$ with one degree of freedom chi-square test, respectively. The threshold values of 2 and 10\% from chi-square test represent approximately 1 and 5\% significant levels of $\mathrm{F}$ test, respectively. 


\subsection{QTL position and heritability}

The QTL positions and heritabilities from five combinations of population, trait and chromosome, which displayed significant levels using all methods, are shown in Table II.

Two combinations showed slightly different peaks between the two methods. The peaks for DGW from population B were 25 and $28 \mathrm{cM}$ on chromosome 4 and the peaks for BFT from population C were 35 and $39 \mathrm{cM}$ on chromosome 7. However, the peaks obtained from the two methods were always bracketed by the same pair of markers. The curves of the QTL test statistic on test positions were shown in Figure 2 for three traits as examples.

The polygenic heritability, $h_{p}^{2}\left(=\sigma_{p}^{2} /\left(\sigma_{p}^{2}+\sigma_{q}^{2}+\sigma_{e}^{2}\right)\right)$, and the QTL genotypic heritability, $h_{q}^{2}\left(=\sigma_{q}^{2} /\left(\sigma_{p}^{2}+\sigma_{q}^{2}+\sigma_{e}^{2}\right)\right)$, were estimated at the estimated QTL position. The largest difference between QTL heritabilities from SMD and MCMC was for DGW on chromosome 4 from population B, where the estimates were $14.0 \%$ and $8.6 \%$, respectively.

\subsection{QTL genotypic and allelic effect}

Using IBD matrices obtained from the SMD method, the QTL genotypic effect at the peak position was converted into allelic effects. The genotypic and allelic effects of sires for five combinations are shown in Table III. Some combinations had a large range of genotypic effects across sires. For example, the ranges of genotypic effect for DGT and BFT from population A were $112.6 \mathrm{~g}$ $(=62.5-(-50.1))$ and $1.77 \mathrm{~mm}(=0.80-(-0.97))$, respectively. These values are $10.9 \%$ and $19.5 \%$, respectively, of the appropriate phenotypic mean. The range of genotypic effects in a population varied from $4.8 \%$ to $10.9 \%$ of the phenotypic mean for growth traits and $7.9 \%$ to $19.5 \%$ for BFT.

In a marker-assisted selection scheme, the differences between QTL allelic effects within a sire are important, since a choice of one of the two QTL alleles related to the marker has a large effect on the genetic performance of the progeny. Some sires had substantially different values for the estimates of the two allelic effects. For example, for DGT from population A, sire 1 had two allelic effects, -58.7 and $17.3 \mathrm{~g}$, which conferred the largest difference, $76 \mathrm{~g}$ $(=17.3-(-58.7))$, across sires in population A. The two other sires, 2 and 10, also displayed significantly divergent values of $56 \mathrm{~g}$ and $45 \mathrm{~g}$, respectively. LS analyses on these three sires revealed the most significant t-values [22]. Across the populations, sires with a significant QTL effect estimated with LS generally displayed considerably divergent estimates of allelic effects in VCA.

A joint dataset comprising data from all five populations were analysed using the SMD and MCMC VCA methods. Only BFT on chromosome 7, analysed by the MCMC method reached the significance level (10\%). In this case, 
Y. Nagamine et al.

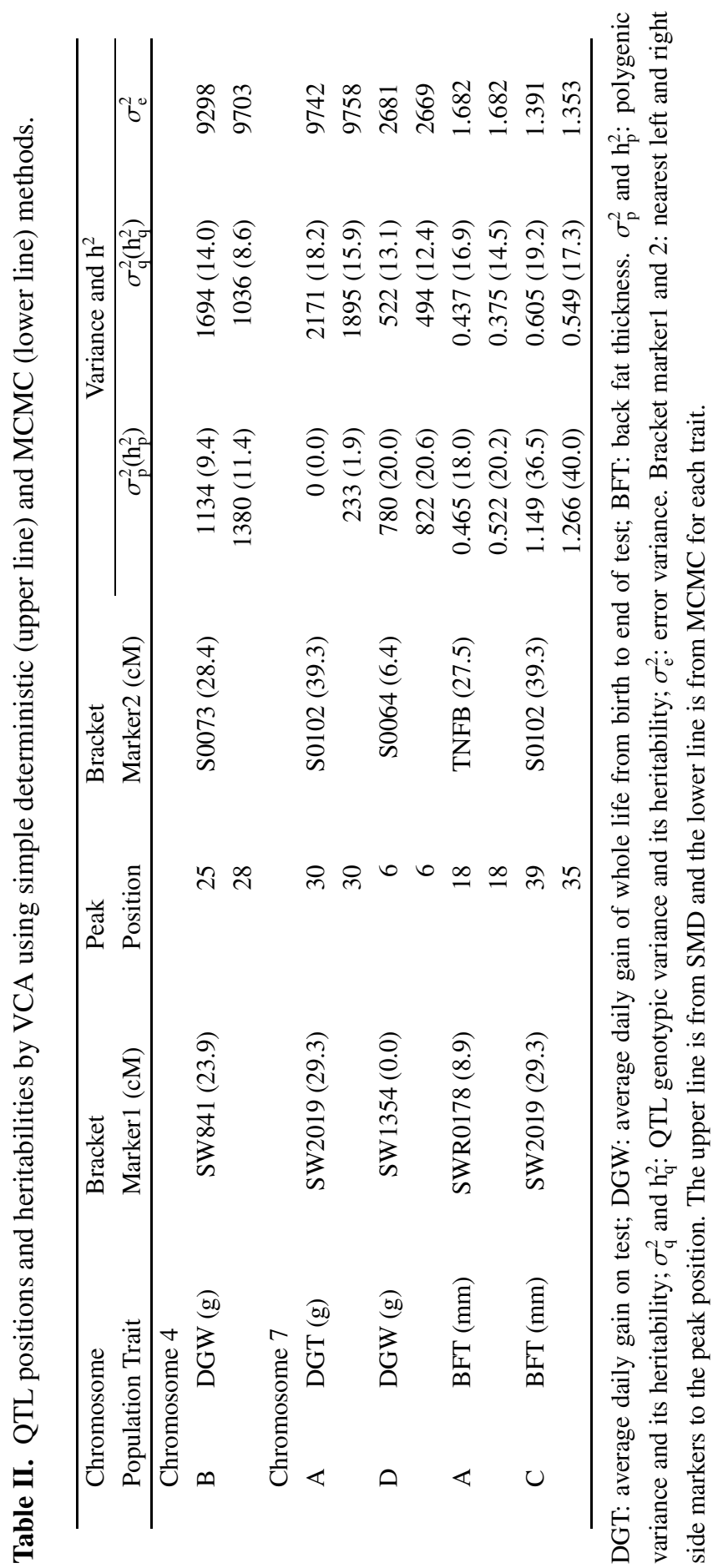



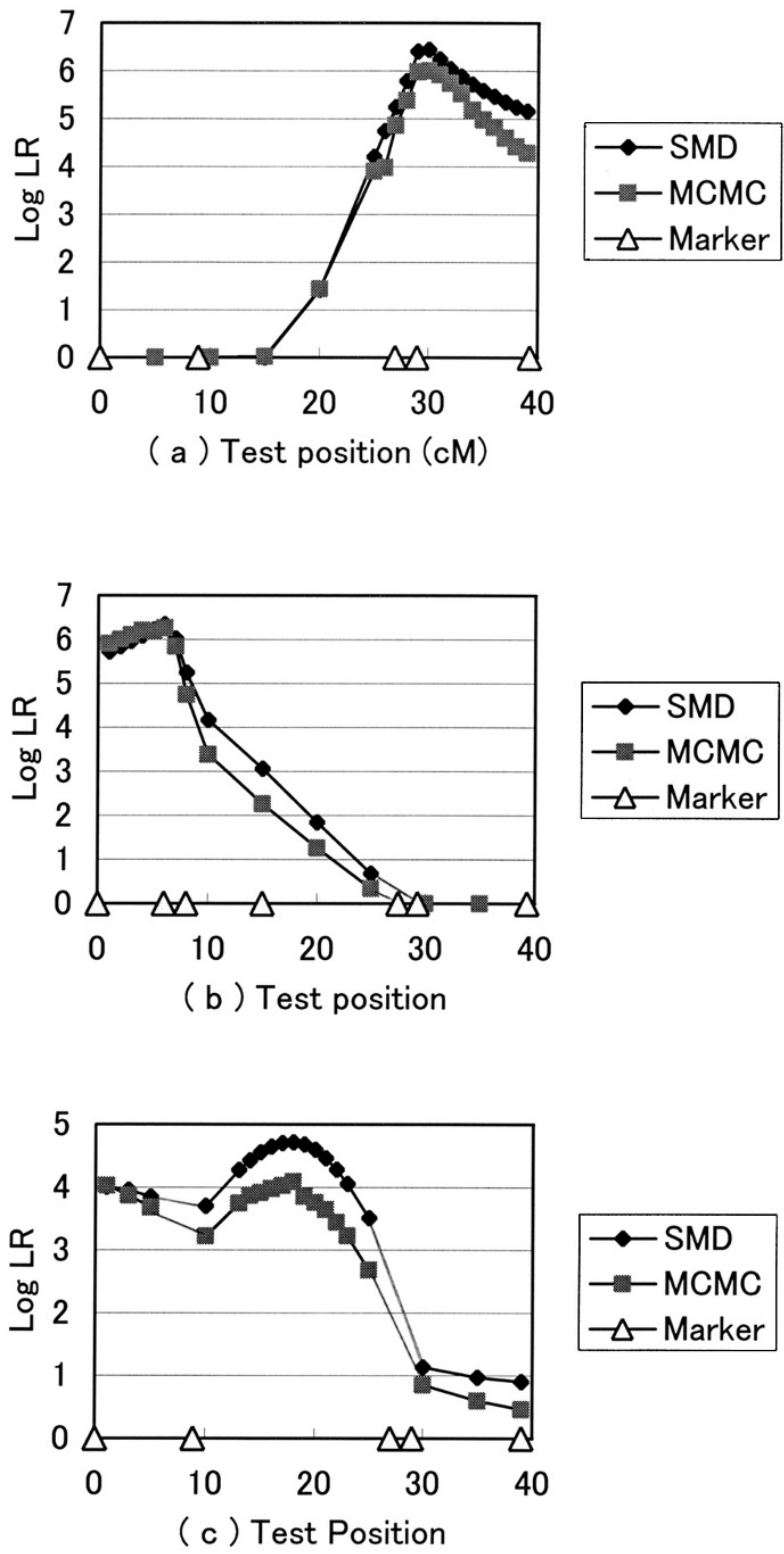

Figure 2. (a) QTL position for DGT, daily gain on test, using chromosome 7 from population A. (b) QTL position for DGW, whole life time daily gain, using chromosome 7 from population D. (c) QTL position for BFT, back fat thickness, using chromosome 7 from population A. Seven markers SW1354, S0064, SWR1078, SW1344, $T N F B, S W 2019$ and $S 0102$ (from left to right) were used for population D and 5 markers, excluding SO064 and SW1344, were used for population A. 
Table III. QTL genotypic and allelic effect of sires by VCA using simple deterministic (SMD) method.

\begin{tabular}{|c|c|c|c|c|c|c|c|}
\hline \multirow{2}{*}{\multicolumn{2}{|c|}{$\begin{array}{l}\text { Chromosome } \\
\text { Population Trait }\end{array}$}} & \multirow{2}{*}{$\begin{array}{l}\text { Phenotypic } \\
\text { mean }\end{array}$} & \multicolumn{2}{|c|}{ Genotypic effect (SE) } & \multicolumn{3}{|c|}{ Allelic effect } \\
\hline & & & Min & Max & Min & Max & MaxDiff \\
\hline \multicolumn{8}{|c|}{ Chromosome 4} \\
\hline B & $\operatorname{DGW}(\mathrm{g})$ & 640 & $-26.8(33.2)$ & $41.2(33.1)$ & -31.4 & 51.0 & 60.7 \\
\hline \multicolumn{8}{|c|}{ Chromosome 7} \\
\hline A & $\operatorname{DGT}(\mathrm{g})$ & 1034 & $-50.1(25.3)$ & $62.5(34.2)$ & -58.7 & 53.9 & 76.0 \\
\hline $\mathrm{D}$ & $\operatorname{DGW}(\mathrm{g})$ & 642 & $-15.9(19.7)$ & $15.1(19.7)$ & -17.6 & 26.3 & 38.3 \\
\hline A & $\mathrm{BFT}(\mathrm{mm})$ & 9.05 & $-0.97(0.52)$ & $0.80(0.52)$ & -0.56 & 0.78 & 0.97 \\
\hline $\mathrm{C}$ & $\mathrm{BFT}(\mathrm{mm})$ & 8.64 & $-0.25(0.62)$ & $0.43(0.62)$ & -0.49 & 0.55 & 0.78 \\
\hline
\end{tabular}

DGT: average daily gain on test; DGW: average daily gain of whole life from birth to end of test; BFT: back fat thickness. Min and Max: maximum and minimum estimated values across sires within each population. MaxDiff of allelic effects: the maximum difference between two allelic effects in the sire. For example, Sire 1 for DGT in population A had two allelic effects, -58.7 and $17.3 \mathrm{~g}$, and it gave the largest range, $76 \mathrm{~g}(=17.3-(-58.7))$, across sires in population A.

the test statistic surface was relatively flat and the peak for BFT was not very clear. The minimum test statistic was 2.2 between $20 \mathrm{cM}$ and $38 \mathrm{cM}$ and, the maximum value was only 3.0 at 26 and $27 \mathrm{cM}$.

\section{DISCUSSION}

In this study we have detected QTL segregating in several outbred commercial pig populations and estimated the QTL heritabilities and the associated genotypic effects. Following studies by Fernando and Grossman [7], showing how information on a single marker-linked QTL could be incorporated into the mixed model equations, several simulation studies of marker-assisted selection were reported $[20,26]$. The efficiency of using marker information is dependent on several factors, such as QTL allelic effect and recombination rates between QTL and markers. These parameters have not been reported within outbred pig populations. In fact, prior to reports on QTLs within closed populations [5,22], the issue of whether QTL would be found within a modern pig population was a matter of debate. Studies such as the one reported here will help to resolve this debate.

Meuwissen and Goddard [20] showed that a change in the recombination rate from 0.05 to 0.2 was accompanied by a decrease in genetic gain from marker-assisted selection of $7.7 \%$ in simulation. Recombination rates of 0.05 and 0.2 correspond to $5.3 \mathrm{cM}$ and $25.5 \mathrm{cM}$ in a Haldane map distance [12]. In our study, the average distances between markers were $4.1 \mathrm{cM}$ and $6.6 \mathrm{cM}$ 
for chromosome 4 and 7, respectively. Such small distances between markers can increase the genetic gain in practice. Spelman and Bovenhuis [26] assumed QTL heritabilities of 5 and $10 \%$. These values are moderate, compared with our results. In our study, there is a possibility of overestimation of heritability for growth traits [19], since the best and worst progeny groups with respect to growth rate within the sire family were genotyped. However, BFT was not expected to be significantly influenced by selective genotyping [22]. The minimum heritability for BFT was $14.5 \%$ (Tab. II). The results reported here are thus within the range used in simulation studies and suggest some optimism for the eventual application of marker-assisted selection. However, since all estimated variance components from our data set are prone to large standard errors, a larger number of animals and a more complicated model, e.g., including QTL dominance effect, may be required to estimate more reliable parameters for breeding plans.

The confidence interval for QTL position is also important in practice to determine the breeding strategy [30]. However, bootstrapping cannot be easily applied to variance component analysis with general pedigrees and the LOD drop-off method is likely to be biased in real data sets $[19,28,30]$. The further study is required to develop good estimates of confidence intervals for VCA.

There are a few reports on QTL within a closed pig population [5,22]. However, several studies have focused on the detection of QTL on chromosome 4 and 7 using breed crosses [2, 4, 18]. Bidanel et al. [3] studied a Meishan and Large White cross and reported highly significant QTL effects for growth traits between markers, SLA and S0102, on chromosome 7. This position is very close to our detected position from population A. Wang and colleagues [31] also determined the QTL for growth rate on chromosome 7, using Chinese and European crosses. de Koning et al. [4] reported a highly significant QTL for back fat around the marker, S0102, where the QTL was identified in population $\mathrm{C}$ in our study. It is not surprising that large-effect genes for growth trait are located on the same chromosome across the breeds. However, the main issue that requires clarification is how QTL alleles that have large effects between lines or breeds, are maintained within modern pig populations. A number of hypotheses have been put forward to explain this phenomenon. For example, pleiotropy, i.e., a gene having more than one phenotypic effect [6], may aid in the maintenance of QTL alleles with effects in different directions on two traits.

VCA had some additional advantages compared with LS analysis, as the QTL genotypic and allelic effects could be determined with this method when the QTL effect was significant. The heritabilities of polygenic effects and of QTL effects were also estimated simultaneously. These advantages can be obtained from both the SMD and MCMC approaches, and the results from the 
two approaches correlated well. However, the MCMC approach is computationally intensive, as it required a large number of iterations, especially when the QTL test position was close to the marker. These results, showing the detection of QTL within commercial populations and the estimation of their effects with a computationally tractable approach is encouraging for the use of marker-assisted selection. However, more data are required to estimate reliable parameters across a range of traits and hence to help elucidate the reason for the presence of a QTL with large effects within a heavily selected population before marker-assisted selection can be applied in the confident knowledge that there will be no unforeseen side effects.

\section{ACKNOWLEDGEMENTS}

We thank the commercial partners, Cotswold, JSR Healthbred, PIC International, Rattlerow Ltd and Newsham Ltd for generous support in supplying blood or tissue samples and phenotypic information. This project was funded by the Biotechnology and Biological Sciences Research Council under the Sustainable Livestock Production LINK program.

\section{REFERENCES}

[1] Almasy L., Blangero J., Multipoint quantitative-traits linkage analysis in general pedigrees, Am. J. Hum. Genet. 62 (1998) 1198-1211.

[2] Andersson L., Haley C.S., Ellegren H., Knott S.A., Johansson M., Andersson K., Andersson-Eklund L., Edfors-Lilja I., Fredholm M., Hasson I., Hakansson J., Lundström K., Genetic mapping of quantitative trait loci for growth and fatness in pigs, Science 263 (1994) 1771-1774.

[3] Bidanel J.P., Milan D., Iannuccelli N., Amigues Y., Boscher M.Y., Bourgeois F., Caritez J.C., Gruand J., Le Roy P., Lagant H., Quintanilla R., Renard C., Gellin J., Ollivier L., Chevalet C., Detection of quantitative trait loci for growth and fatness in pigs, Genet. Sel. Evol. 33 (2001) 289-309.

[4] de Koning D.J., Janss L.L.G., Rattink A.P., Van Oers P.A.M., De Vries B.J., Groenen M.A.M., Van Der Poel J.J., De Groot P.N., Brascamp E.W., Van Arendonk J.A.M., Detection of quantitative traits loci for back fat thickness and intramuscular fat content in pigs (Sus scrofa), Genetics 152 (1999) 1679-1690.

[5] de Koning D.J., Nagamine Y., Evans G., Haley C.S., QTL detection in outbred populations, in: Proceedings of the 7th World Congress on Genetics Applied to Livestock Production, Montpellier, August 19-23, 2002, INRA, CastanetTolosan, pp. 657-660.

[6] Falconer D.S., Mackay T.F.C., Introduction of quantitative genetics, 4th edn., Longman limited, London, 1996. 
[7] Fernando R.L., Grossman M., Marker assisted selection using best linear unbiased prediction, Genet. Sel. Evol. 21 (1989) 467-477.

[8] George A.W., Visscher P.M., Haley C.S., Mapping quantitative trait loci in complex pedigrees, Genetics 156 (2000) 2081-2092.

[9] Gilmour A.R., Cullis B.R., Welham S.J., Thompson R., ASREML. Program user manual. Orange agricultural Institute, NSW, 1999.

[10] Green P., Falls K., Crooks S., Documentation for CRIMAP version 2.4, Washington University School of Medicine, Saint Louis, 1990.

[11] Grignola F.E., Hoeschele I., Tier B., Mapping quantitative trait loci in outcross populations via residual maximum likelihood. I. Methodology, Genet. Sel. Evol. 28 (1996) 479-490.

[12] Haldane J.B.S., The combination of linkage values, and the calculation of distances between the loci of linked factors, J. Genet. 8 (1919) 299-309.

[13] Haley C.S., Knott S.A., A simple method for mapping quantitative trait loci in line crosses using flanking markers, Heredity 69 (1992) 315-324.

[14] Haley C.S., Knott S.A., Elsen J.M., Mapping quantitative trait loci in crosses between outbred lines using least squares, Genetics 136 (1994) 1195-1207.

[15] Heath S.C., Markov chain Monte Carlo segregation and linkage analysis for oligogenic models, Am. J. Hum. Genet. 61 (1997) 748-760.

[16] Henderson C.R., Application of linear models in animal breeding, 1st edn., University of Guelph, Ontario, 1984.

[17] Knott S.A., Elsen J.M., Haley C.S., Method for multiple-marker mapping of quantitative trait loci in half-sib populations, Theor. Appl. Genet. 93 (1996) 71-80.

[18] Knott S.A., Marklund L., Haley C.S., Andersson K., Davies W., Ellegren H., Fredholm M., Hansson I., Hoyhem B., Lundström K., Moller M., Andersson L., Multiple marker mapping of quantitative trait loci in a cross between outbred wild boar and large white pigs, Genetics 149 (1998) 1069-1080.

[19] Martinez M.L., Vukasinovic N., Freeman A.E., Fernando R.L., Mapping QTL in outbred populations using selected samples, Genet. Sel. Evol. 30 (1998) 453-468.

[20] Meuwissen T.H.E., Goddard M.E., The use of marker haplotypes in animal breeding schemes, Genet. Sel. Evol. 28 (1996) 161-176.

[21] Nagamine Y., Knott S.A., Visscher P.M., Haley C.S., Simple deterministic identity-by-descents coefficients and estimation of QTL allelic effects in full and half sibs, Genet. Res. 80 (2002) 237-243.

[22] Nagamine Y., Haley C.S., Sewalem A., Visscher P.M., Quantitative trait loci variation for growth and obesity between and within lines of pigs (Sus scrofa), Genetics 164 (2003) 629-635.

[23] Pong-Wong R., George A.W., Woolliams J.A., Haley C.S., A simple and rapid method for calculating identity-by-descent matrices using multiple markers, Genet. Sel. Evol. 33 (2001) 453-471.

[24] Rohrer G.A., Keele J.W., Identification of quantitative trait loci affecting carcass composition in swine. I. Fat deposition traits, J. Anim. Sci. 76 (1998) 2247-2254. 
[25] Sorensen A.C., Pong-Wong R., Windig J., Woolliams J.A., Precision of methods for calculating identity-by-descent matrices using multiple markers, Genet. Sel. Evol. 34 (2002) 557-579.

[26] Spelman R.J., Bovenhuis H., Genetic response from marker assisted selection in an outbred population for differing marker bracket sizes and with two identified quantitative trait loci, Genetics 148 (1998) 1389-1396.

[27] Van Arendonk J.A.M., Tier B., Kinghorn B.P., Use of multiple genetic markers in prediction of breeding values, Genetics 137 (1994) 319-329.

[28] van Ooijen J.W., Accuracy of mapping quantitative trait loci in autogamous species, Theor. Appl. Genet. 84 (1992) 803-811.

[29] Visscher P.M., Haley C.S., Utilizing genetic markers in pig breeding programmes, Anim. Breed. Abstr. 63 (1995) 1-8.

[30] Visscher P.M., Haley C.S., Detection of putative quantitative trait loci in crosses under infinitesimal genetic models, Theor. Appl. Genet. 93 (1996) 691-702.

[31] Wang L., Yu T.P., Tuggle C.K., Liu H.C., Rothschild M.F., A directed search for quantitative trait loci on chromosome 4 and 7 in pigs, J. Anim. Sci. 76 (1998) 2560-2567.

[32] Xu S., Atchley W.R., A random model approach to interval mapping of quantitative trait loci, Genetics 141 (1995) 1189-1197.

To access this journal online: www.edpsciences.org 